\title{
Conductivity bound of the strongly interacting and disordered graphene from gauge/gravity duality
}

\author{
Marek Rogatkd* and Karol I. Wysokińsk \\ Institute of Physics \\ Maria Curie-Sklodowska University \\ 20-031 Lublin, pl. Marii Curie-Sklodowskiej 1, Poland
}

(Dated: February 7, 2020)

\begin{abstract}
The carriers in graphene tuned close to the Dirac point envisage signatures of the strongly interacting fluid and are subject to hydrodynamic description. The important question is whether strong disorder induces the metal - insulator transition in this two-dimensional material. The bound on the conductivity tensor found earlier within the single current description, implies that the system does not feature metal - insulator transition. The linear spectrum of the graphene imposes the phase - space constraints and calls for the two - current description of interacting electron and hole liquids. Based on the gauge/gravity correspondence, using the linear response of the black brane with broken translation symmetry in Einstein-Maxwell gravity with the auxiliary $U(1)$-gauge field, responsible for the second current, we have calculated the lower bound of the DC-conductivity in holographic model of graphene. The calculations show that the bound on the conductivity depends on the coupling between both $U(1)$ fields and for a physically justified range of parameters it departs only weakly from the value found for a model with the single $U(1)$ field.
\end{abstract}

\section{INTRODUCTION}

Disorder and interactions inside solids are responsible for finite values of the transport coefficients and play a very important role in establishing their detailed behavior. Importantly, the role of both disorder and interactions depends on the spatial dimensionality of the condensed matter system. Doping of the intrinsic semiconductors, being the key ingredient of numerous electronic applications, is an important example illustrating the role of disorder in the weakly interacting three-dimensional materials.

It has been predicted and verified experimentally that in three-dimensional systems both strong disorder or strong electron-electron interactions can induce metal to insulator transition. In the non-interacting systems this phenomenon is called the Anderson transition [1], while in the presence of electron-electron interaction, the transition is known as Mott [2] or if interactions and disorder play a significant role, the Anderson-Hubbard one [3].

On the other hand, the two-dimensional systems are far more complicated from the experimental [4 6 ] , as well as, the theoretical points of view. The theoretical description of the interacting 7] systems in question, does not give unique results. The recent application of the gauge/gravity analogy to study the strongly interacting two-dimensional disordered materials has revealed the absence of the disorder driven metal-insulator transition in the system [8]. The result is valid in the hydrodynamic limit for the electron mean free path much smaller than the typical scale of the spatial inhomogeneities [9].

The hydrodynamic limit of the electron flow has been identified experimentally in very clean systems, as predicted long time ago 10]. In fact, the signatures of the hydrodynamic behavior have been observed over the last years in many materials including the high mobility $(\mathrm{Al}, \mathrm{Ga})$ As wires [11, 12]. More recent measurements have envisaged the hydrodynamic signatures in many other materials. One should mention the shear viscosity measurements in the ultra-cold Fermi gases [13], strongly correlated oxides [14] and graphene [15, 16]. The comprehensive discussion of this novel set of experiments is given in [17].

The special interest is devoted to graphene, the two-dimensional system which envisages a hydrodynamic behavior of the carriers, observed in a number of recent experiments [18 20, 25], especially when the material is tuned close to the particle-hole symmetry point. Due to the strong scattering of charge carriers, in the nearby of the charge neutrality point, the thermoelectric power of graphene is strongly enhanced [18] and approaches the hydrodynamic limit. The departures from the Wiedemann-Franz ratio due to the increase of thermal conductivity [16], have been interpreted as the indication of hydrodynamic behavior in material in question. On the other hand, the hydrodynamic viscosity of electrons has been measured [15] in a high mobility graphene samples. The viscous effects were observed [19] and

*Electronic address: marek.rogatko@poczta.umcs.lublin.pl, rogat@kft.umcs.lublin.pl

${ }^{\dagger}$ Electronic address: karol.wysokinski@poczta.umcs.pl 
shown to facilitate high mobility transport at temperatures below $150 \mathrm{~K}$. The recent theoretical and experimental studies of hydrodynamic effect in graphene have been reviewed in [21, 22].

Even though the hydrodynamic flow is expected to be observed in a very clean system, the disorder seems to be an important factor which sometimes even facilitates the hydrodynamic behavior [23]. The signatures of the Stokes non-linear flow with the low Reynolds number 24] have been detected in graphene [25], as the appearance of vortices leading to the negative resistance of the material.

At the Fermi level graphene exhibits a massless relativistic spectrum with Dirac cone. As was mentioned above, close to the charge neutrality point, it sustains a strongly interacting material, ideal system for studies by means of gauge/gravity duality methods. In this system, the thermoelectric transport coefficients have been found using the hydrodynamic approach [26 28], with a fairly good agreement with the experimental data.

Recently, this attitude has been generalized to the model with two distinct $U(1)$-gauge currents, which is solved by the AdS/CFT analogy [29]. The model in question allowed the successful quantitative comparison between theory and experimental data. The paper [29] gives a number of arguments behind the introduction of two gauge fields and associated currents. One reason for the appearance of two currents in graphene is the charge imbalance between electrons and holes in the system with linear spectrum. It has been found that the two current model allows for a quantitatively correct description of the thermal conductance of graphene. The paper 30] presented the further generalization, taking into account the possible coupling between both currents. In Ref. [30] the transport properties of graphene using the model in question were elaborated. Moreover the perpendicular magnetic field to the graphene sheet was taken into account. It was assumed that the charges bounded with the two gauge fields are proportional to each other with the factor $g$, which is responsible for the possible imbalance of the positive and negative charges in graphene close to the charge neutrality point. It was found that the kinetic and transport coefficients were influenced by $\alpha$-coupling constant and factor $g$. The increase of $\alpha$ leads to the increase of the width of normalized thermal conductivity, while in the case when $g=0$, the effect has been quite opposite (we have the decrease of the width). On the other hand, the $\alpha$-coupling constant affects Wiedeman-Franz ratio (WFR), changing the width and heights of the curves. The general tendency is that WFR diminishes while the value of $\alpha$-coupling constant grows.

Moreover the coupling constant in question, impels the charge dependence of the diagonal resistivity and the WFR, i.e., the increase of $\alpha$ causes the decrease of both $\rho^{x x}$ and $W^{x x}$. The Seebeck and Nernst coefficients were affected by magnetic field and $\alpha$. The influence in question, for large value of $S^{x x}$, changes the shape of the curve from two minima and a maximum curve to the one with a minimum (for $B=0$ ) and two small maxima for larger absolute values. The Hall angle was also influenced by the coupling constant. In the studied case the density dependence of the thermoelectric coefficient $\alpha_{i j}$ and Seebeck coefficient $S^{x x}$ agree with experimental data.

The generalizations of these researches were given in [31], where the holographic calculation of magneto transport coefficients in $3+1$ dimensional system with Dirac-like spectrum were presented. The calculations envisage the influence of $g$ and $\alpha$ on the coefficients. Namely, the magnetic field dependence on resistivity $\rho^{x x}$ and $\rho^{x y}$ depicts that the bigger values of $\alpha$ one takes the smaller resistivity we achieve.

In general one expects the presence of additional gauge fields in graphene due to geometric and other reasons [32, 33]. The use of gauge/gravity duality allows for the exact solution of the strongly coupled field theoretical models. We use this approach to elaborate the effect of interactions and disorder on the hydrodynamic transport of graphene modeled by the $3+1$ dimensional AdS space time with the black brane background which breaks translational symmetry [34, 35].

The studies of electrical transport in a strongly coupled system include the case of strange metals in two spatial dimensions at finite temperature and charge density, holographically dual to Einstein-Maxwell theory with a potential in asymptotically four-dimensional AdS manifold. One finds that the electrical conductivity is bounded from below by a universal minimum conductance. The inspection of Stokes-like equations in the spacetime in question shows that it cannot exhibit metal-insulator transitions [8]. The bound on the incoherent thermal conductivity obtained by analyzing the linear perturbations of black brane with broken translation symmetry in AdS Einstein-Maxwell dilaton gravity was performed in [36]. It turns out that the thermal conductivity has non-zero value (at finite temperature), as far as, the dilaton potential is bounded from below.

In 37] the analytical lower bound on the conductivity in holographic model AdS Einstein-Maxwell dilaton theory, in terms of black horizon data, using the Stokes equations on black object event horizon was provided. In the considered model the metal-insulator transition is not driven by disorder, but it is caused by coupling scalar field to Maxwell one. Studies in rotational and translational symmetries breaking system reveal that the ratio of the determinant of the electrical conductivities along any spatial directions, to black brane area density, having the zero charge limit in account, tends to the universal value [38]. The conductivity bounds were also elaborated in the case of probe brane models [39], massive gravity [40], as well as, in effective holographic theories [41]. It was shown in the two latter cases that there were no bounds on conductivities.

In [43] the Navier-Stokes equations of the model with two $U(1)$-gauge fields were derived. The paper elaborates the black brane response to the electric fields and temperature gradient. The DC transport coefficients for the holographic Dirac semimetals are found. Here we analyze similar model with a goal to establish the bounds on the conductivity 
of the Dirac fluid in graphene subject to the influence of $\alpha$-coupling constant between the two $U(1)$-gauge fields. The main result is that the coupling between the currents, in the following quantified by the parameter $\alpha$ only slightly modifies the bounds (see Eqs. (63) and (85)) on the conductivity for $\alpha \leq 1$. Larger values of $\alpha$ lead to strong decrease of the bound and finally to metal - insulator transition at $\alpha=2$, when the conductivity bound vanishes.

The organization of the paper is as follows. In Sec. [1] we introduce the gravitational background and the action used to describe two interacting currents in graphene. Sec. III is devoted to the description of the perturbations of the event horizon allowing the derivation of the appropriate hydrodynamic description. We calculate the conductivity of the system in the background of the uncharged black brane in Sec. IV] In section $\nabla$ the case of the charged background black brane is discussed, where we also derive the lower bounds on the conductivity. The variational approach has been applied in Sec. VI to study the conductivity bounds. In Sec. VII we end up with the summary and conclusions.

\section{BACKGROUND HOLOGRAPHIC MODEL}

In our paper we deal with the generalization of the previously studied models [34, 35], by adding two interacting $U(1)$-gauge fields. The aim is to find the influence of them on DC thermoelectric transport coefficients and to compare with the existing results. In our model the gravitational action in $(3+1)$-dimensions is taken in the form

$$
S=\int \sqrt{-g} d^{4} x\left(R+\frac{6}{L^{2}}-\frac{1}{2} \nabla_{\mu} \phi \nabla^{\mu} \phi-\frac{1}{4} F_{\mu \nu} F^{\mu \nu}-\frac{1}{4} B_{\mu \nu} B^{\mu \nu}-\frac{\alpha}{4} F_{\mu \nu} B^{\mu \nu}\right),
$$

where $R$ is the scalar curvature of the spacetime, $\phi$ stands for the scalar field, which as we shall see later on contributes a viscosity like term to the hydrodynamic equations. $F_{\mu \nu}=2 \nabla_{[\mu} A_{\nu]}$ are ordinary Maxwell field strength tensor, while the second $U(1)$-gauge field $B_{\mu \nu}$ is given by $B_{\mu \nu}=2 \nabla_{[\mu} B_{\nu]} . \alpha$ is the coupling constant between both gauge fields. $L$ is the radius of AdS-spacetime.

The presence of additional gauge field is motivated by the desire of describing carrier flow in graphene, near the particle-hole symmetry point. These two currents may be interpreted as connected with electrons and holes. The approach in question provides quantitatively correct description of the thermal conductivity of graphene close to the Dirac point [29]. Allowing for the interaction between the two $U(1)$-gauge currents, the coupling $\alpha$ provides additional degree of freedom and inter alia affects [30] the magnetic field dependence of the non-diagonal transport coefficients, especially for the low values of the aforementioned field. The important novel aspect of the two current model is the tensor structure [29, 30] of the transport coefficients with the general entries, e.g., for the conductivity $\sigma_{a b}^{i j}$, where $a, b$ refer to two fields denoted above as $F$ and $B$ and $i, j$ refer to the spatial directions (c.f. Eqs. (53) and (54)). The identifications of the charges $Q_{F}=-e n_{e}$ and $Q_{B}=+e n_{h}$ with electrons and holes and the total electric current $J^{j}=J_{F}^{j}+J_{B}^{j}$ as well as assuming that the electric fields $E_{F}^{i}=E_{B}^{i}=E^{i}$ lead to the value of the total conductivity elements $\sigma^{i j}=\sum_{a, b} \sigma_{a b}^{i j}$. The presence of the coupling $\alpha$ between the fields leads to non-zero values of $\sigma_{F B}^{i j}$. Moreover, independently whether the coupling vanishes or not it is important to keep the tensor structure of the kinetic and transport coefficients [29, 30]. The analogous studies of the magneto-transport coefficients of Dirac semimetals [31], being the three-dimensional analogues of graphene require similar treatment of the conductivity. In both cases, in order to define the other transport coefficients, like thermoelectric tensor or Hall angle, one needs to take the full tensorial character of the conductivity into consideration.

In the studied action (11) we have to do with the second gauge field coupled to the ordinary Maxwell one. The justifications of such kind of gravity with electromagnetism coupled to the other gauge field follow from the topdown perspective [42]. Namely, starting from the string/M-theory the reduction to the lower dimensional gravity is performed. It is relevant in the holographic correspondence attitude, because the theory in question is a fully consistent quantum theory (string/M-theory) and this fact guarantees that any predicted phenomenon by the top-down theory will be physical. This point has been discussed in [43].

Variation of the action $S$ with respect to the metric, the scalar and gauge fields yields the following equations of motion:

$$
\begin{aligned}
G_{\mu \nu} & -g_{\mu \nu} \frac{3}{L^{2}}=T_{\mu \nu}(\phi)+T_{\mu \nu}(F)+T_{\mu \nu}(B)+\alpha T_{\mu \nu}(F, B), \\
\nabla_{\mu} F^{\mu \nu} & +\frac{\alpha}{2} \nabla_{\mu} B^{\mu \nu}=0, \\
\nabla_{\mu} B^{\mu \nu} & +\frac{\alpha}{2} \nabla_{\mu} F^{\mu \nu}=0, \\
\nabla_{\mu} \nabla^{\mu} \phi & =0
\end{aligned}
$$

where we have denoted by $G_{\mu \nu}$ the Einstein tensor, while the energy momentum tensors for the fields in the theory 
are given by

$$
\begin{aligned}
T_{\mu \nu}(\phi) & =\frac{1}{2} \nabla_{\mu} \phi \nabla_{\nu} \phi-\frac{1}{4} g_{\mu \nu} \nabla_{\delta} \phi \nabla^{\delta} \phi \\
T_{\mu \nu}(F) & =\frac{1}{2} F_{\mu \delta} F_{\nu}^{\delta}-\frac{1}{8} g_{\mu \nu} F_{\alpha \beta} F^{\alpha \beta} \\
T_{\mu \nu}(B) & =\frac{1}{2} B_{\mu \delta} B_{\nu}^{\delta}-\frac{1}{8} g_{\mu \nu} B_{\alpha \beta} B^{\alpha \beta} \\
T_{\mu \nu}(F, B) & =\frac{1}{2} F_{\mu \delta} B_{\nu}^{\delta}-\frac{1}{8} g_{\mu \nu} F_{\alpha \beta} B^{\alpha \beta} .
\end{aligned}
$$

For the gauge fields in the considered theory we assume the following components:

$$
A_{\mu} d x^{\mu}=a_{t} d t, \quad B_{\mu} d x^{\mu}=b_{t} d t
$$

\section{PERTURBATIONS OF BACKGROUND BLACK BRANE}

In the following analysis we consider the line element provided by

$$
d s^{2}=-U(r) G\left(r, x_{i}\right) d t^{2}+\frac{F\left(r, x_{i}\right) d r^{2}}{U(r)}+d s^{2}\left(\Sigma_{2}\right),
$$

where $\Sigma_{2}$ stands for the two-dimensional hypersurface at chosen radial $r$-coordinate. The dependence of function $G$ and $F$ on the $x_{i}$ coordinates takes care of their spatial variations. We also take the following components of the fields

$$
A=a_{t} d t, \quad B=b_{t} d t
$$

As in [34], the line element at $r \rightarrow \infty$ approaches the AdS boundary with the following conditions:

$$
\begin{array}{r}
U \rightarrow r^{2}, \quad F \rightarrow 1, \quad G \rightarrow G(x), \quad g_{i j} \rightarrow r^{2} \bar{g}_{i j} \\
a_{t}\left(r, x_{i}\right) \rightarrow \mu(x), \quad b_{t}\left(r, x_{i}\right) \rightarrow \mu_{d}(x), \quad \phi\left(r, x_{i}\right) \rightarrow r^{\Delta-3} \bar{\phi}\left(x_{i}\right),
\end{array}
$$

where $\mu(x)$ and $\mu_{d}(x)$ are the spatially dependent chemical potentials (at the boundary) connected with the adequate $U(1)$-gauge field. We also assume the periodic boundary conditions with period $L_{i}$ in the i-th direction: $f\left(x_{i}+L_{i}\right)=$ $f\left(x_{i}\right)$ and if required shall work with quantities averaged over the volume of periodicity $\mathbb{E}[f]=\frac{1}{L_{x_{1}} L_{x_{2}}} \int d x_{1} d x_{2} f$. $\bar{\phi}\left(x_{i}\right)$ above serves as a boundary source of the field $\phi\left(r, x_{i}\right)$ and $\Delta$ is the scaling dimension of it.

The black brane event horizon which has $\Sigma_{2}$ topology, is situated at $r=0$. Having in mind the Edington-Finkelstein ingoing coordinates, the near-horizon expansions of the metric tensor components and fields are given by [35]

$$
\begin{aligned}
U(r) & =r\left(4 \pi T+U^{(1)} r+\ldots\right), \\
G\left(r, x_{i}\right) & =G^{(0)}(x)+G^{(1)}(x) r+\ldots, \\
F\left(r, x_{i}\right) & =F^{(0)}(x)+F^{(1)}(x) r+\ldots, \\
g_{i j} & =g_{i j}^{(0)}+g_{i j}^{(1)} r+\ldots, \\
a_{t}\left(r, x_{i}\right) & =r\left(a_{t}^{(0)} G^{(0)}(x)+a_{t}^{(1)}(x) r+\ldots\right), \\
b_{t}\left(r, x_{i}\right) & =r\left(b_{t}^{(0)} G^{(0)}(x)+b_{t}^{(1)}(x) r+\ldots\right), \\
\phi\left(r, x_{i}\right) & =\phi^{(0)}(x)+\phi^{(1)}(x) r+\ldots,
\end{aligned}
$$

with the auxiliary condition written as $G^{(0)}(x)=F^{(0)}(x)$.

If one implements the $U(1)$-gauge and temperature gradient in the black brane spacetime, at fixed $r$-coordinate, then the black object will respond. In our considerations we have to take into account linear perturbations described by 35

$$
\begin{aligned}
\delta\left(d s^{2}\right) & =\delta g_{\alpha \beta} d x^{\alpha} d x^{\beta}-2 t M \xi_{a} d t d x^{a}, \\
\delta A & =\delta a_{\beta} d x^{\beta}-t E_{a} d x^{a}+t N \xi_{b} d x^{b} \\
\delta B & =\delta b_{\beta} d x^{\beta}-t B_{a} d x^{a}+t N_{d} \xi_{b} d x^{b}
\end{aligned}
$$


as well as the perturbation of scalar field, $\delta \phi$. In what follows we shall consider $\delta g_{\mu \nu}, \delta a_{\mu}, \delta b_{\mu}$, and $\delta \phi$ as functions of $\left(r, x_{m}\right)$. On the other hand $E_{a}, B_{a}, \xi_{i}$, depend on $x_{i}$-coordinates and are closed forms on $\Sigma_{2}$. Moreover, the regularity at the black brane event horizon implies the following:

$$
\begin{aligned}
\delta g_{t t} & =U(r)\left(\delta g_{t t}^{(0)}\left(x_{i}\right)+\mathcal{O}(r)\right), \quad \delta g_{t r}=\delta g_{t r}^{(0)}\left(x_{i}\right)+\mathcal{O}(r), \\
\delta g_{r r} & =\frac{1}{U(r)}\left(\delta g_{r r}^{(0)}\left(x_{i}\right)+\mathcal{O}(r)\right), \quad \delta g_{i j}=\delta g_{i j}^{(0)}\left(x_{i}\right)+\mathcal{O}(r), \\
\delta g_{t i} & =\delta g_{t i}^{(0)}\left(x_{i}\right)-G U \xi_{i} \frac{\ln r}{4 \pi T}+\mathcal{O}(r), \quad \delta g_{r i}=\frac{1}{U(r)}\left(\delta g_{r i}^{(0)}\left(x_{i}\right)+\mathcal{O}(r)\right), \\
\delta a_{t} & =\delta a_{t}^{(0)}\left(x_{i}\right)+\mathcal{O}(r), \quad \delta a_{i}=\frac{\ln r}{4 \pi T}\left(-E_{i}+N \xi_{i}\right)+\mathcal{O}(r), \\
\delta a_{r} & =\frac{1}{U(r)}\left(\delta a_{r}^{(0)}\left(x_{i}\right)+\mathcal{O}(r)\right), \\
\delta b_{t} & =\delta b_{t}^{(0)}\left(x_{i}\right)+\mathcal{O}(r), \quad \delta b_{i}=\frac{\ln r}{4 \pi T}\left(-B_{i}+N_{d} \xi_{i}\right)+\mathcal{O}(r), \\
\delta b_{r} & =\frac{1}{U(r)}\left(\delta b_{r}^{(0)}\left(x_{i}\right)+\mathcal{O}(r)\right),
\end{aligned}
$$

It turns out that the constraint on the leading order have to be imposed

$$
\begin{aligned}
& \delta g_{t t}^{(0)}+\delta g_{r r}^{(0)}-2 \delta g_{r t}^{(0)}=0, \quad \delta g_{r i}^{(0)}=\delta g_{t i}^{(0)}, \\
& \delta a_{r}^{(0)}=\delta a_{t}^{(0)}, \quad \delta b_{r}^{(0)}=\delta b_{t}^{(0)} .
\end{aligned}
$$

\section{A. Equations for perturbations at the event horizon}

One imposes on a subset of the linearized black brane perturbations, i.e., $\delta g_{i t}^{(0)}, \delta g_{r t}^{(0)}, \delta a_{t}^{(0)}$, $\delta b_{t}^{(0)}$, the relations as follows [43]:

$$
\begin{aligned}
\nabla_{i} \nabla^{i} w & +\nabla_{i} E^{i}+\nabla_{i}\left(a_{t}^{(0)} v^{i}\right)+\frac{\alpha}{2}\left[\nabla_{m} \nabla^{m} w_{d}+\nabla_{m} B^{m}+\nabla_{m}\left(b_{t}^{(0)} v^{m}\right)\right]=0, \\
\nabla_{i} \nabla^{i} w_{d} & +\nabla_{i} B^{i}+\nabla_{i}\left(b_{t}^{(0)} v^{i}\right)+\frac{\alpha}{2}\left[\nabla_{m} \nabla^{m} w+\nabla_{m} E^{m}+\nabla_{m}\left(a_{t}^{(0)} v^{m}\right)\right]=0, \\
b_{t}^{(0)}\left[\nabla_{i} w_{d}\right. & \left.+B_{i}+\frac{\alpha}{2}\left(\nabla_{i} w+E_{i}\right)\right]+a_{t}^{(0)}\left[\nabla_{i} w+E_{i}+\frac{\alpha}{2}\left(\nabla_{i} w_{d}+B_{i}\right)\right] \\
& -\nabla_{i} \phi^{(0)} \nabla_{m} \phi^{(0)} v^{m}+2 \nabla^{m} \nabla_{(m} v_{i)}+4 \pi T \xi_{i}-\nabla_{i} p=0, \\
\nabla_{i} v^{i} & =0
\end{aligned}
$$

where we have denoted

$$
\begin{aligned}
w & =\delta a_{t}^{(0)}, \quad w_{d}=\delta b_{t}^{(0)}, \quad p=-4 \pi T \frac{\delta g_{r t}^{(0)}}{G^{(0)}}-\delta g_{i t}^{(0)} \nabla^{i} \ln G^{(0)}, \\
v_{i} & =-\delta g_{i t}^{(0)} .
\end{aligned}
$$

The above equations result from the conservation of charge and heat currents in the unperturbed system. The variables introduced in (37) are a subset of all perturbations. They are found to fulfill at the horizon the generalized Navier-Stokes equations (33) - (36). As discussed earlier for the single current model [35] the scalar field contributes viscosity like term as also does the curvature of the horizon. The latter is best visible by writing

$$
2 \nabla^{m} \nabla_{(m} v_{j)}=\nabla^{2} v_{j}+R_{j i} v^{i}
$$

\section{CONDUCTIVITY FOR THE UNCHARGED BLACK BRANE}

In this section we shall consider the case without $\phi$ field, responsible for dissipation. Further, for the connectedness with [8] we define the quantities

$$
Q=a_{t}^{(0)}, \quad Q_{d}=b_{t}^{(0)}, \quad \nabla_{j} w=-\nabla_{j} \mu, \quad \nabla_{j} w_{d}=-\nabla_{j} \mu_{d}
$$


Let us first study the conductivities for Dirac semimetals in the uncharged black object case, i.e., $Q=0, Q_{d}=0$. For the considered situation equation (33) and (34) decoupled to the relations

$$
\begin{aligned}
& \nabla_{i}\left[\sqrt{g^{(0)}}\left(E^{i}-\nabla^{i} \mu\right)\right]=0, \\
& \nabla_{i}\left[\sqrt{g^{(0)}}\left(B^{i}-\nabla^{i} \mu_{d}\right)\right]=0 .
\end{aligned}
$$

Because of the fact that they constitute the linear equations, we may set

$$
\mu=\mu_{a} E^{a}, \quad \mu_{d}=\mu_{d_{a}} B^{a},
$$

in the equations (40) and (41). This substitution reveals that

$$
\begin{aligned}
& \nabla_{i}\left[\sqrt{g^{(0)}}\left(\delta_{k}^{i}-\nabla^{i} \mu_{k}\right) E^{k}\right]=0 \\
& \nabla_{i}\left[\sqrt{g^{(0)}}\left(\delta_{k}^{i}-\nabla^{i} \mu_{d_{k}}\right) B^{k}\right]=0 .
\end{aligned}
$$

On the other hand, it follows that for some constants $\psi_{k}^{i},\left(\psi_{d}\right)_{k}^{i}$ and functions $\Psi^{i}, \Psi_{d}^{i}$, one obtains

$$
\begin{aligned}
& \sqrt{g^{(0)}} g_{(0)}^{i j}\left(\delta^{k}{ }_{j}-\nabla_{j} \mu^{k}\right)=\epsilon^{i j}\left(\psi_{k}{ }_{k}-\nabla_{j} \Psi^{k}\right), \\
& \sqrt{g^{(0)}} g_{(0)}^{i j}\left(\delta^{k}{ }_{j}-\nabla_{j} \mu_{d}^{k}\right)=\epsilon^{i j}\left(\left(\psi_{d}\right)_{k}^{i}-\nabla_{j} \Psi_{d}^{k}\right),
\end{aligned}
$$

Using the properties of the antisymmetric two-dimensional tensor $\epsilon^{i j}$, it can be proved, that the above equations are equivalent to

$$
\begin{aligned}
& -\epsilon^{a m}\left(\delta_{m}^{k}-\nabla_{m} \mu^{k}\right)=\sqrt{g^{(0)}} g_{(0)}^{a j}\left(\psi_{j}^{k}-\nabla_{j} \Psi^{k}\right), \\
& -\epsilon^{a m}\left(\delta_{m}^{k}-\nabla_{m} \mu_{d}^{k}\right)=\sqrt{g^{(0)}} g_{(0)}^{a j}\left(\left(\psi_{d}\right)^{k}{ }_{j}-\nabla_{j} \Psi_{d}^{k}\right),
\end{aligned}
$$

Taking the spatial derivatives of the relations (47) and (48), using the uniqueness and linearity arguments, one obtains that

$$
\begin{aligned}
\nabla_{i} \Psi^{k} & =\psi^{k}{ }_{j} \nabla_{i} \mu^{j}, \\
\nabla_{i} \Psi_{d}^{k} & =\left(\psi_{d}\right)^{k}{ }_{j} \nabla_{i} \mu_{d}^{j} .
\end{aligned}
$$

Combining relations (47)-(48) and (49)-(50), we arrive at the following:

$$
\begin{aligned}
& \sqrt{g^{(0)}} g_{(0)}^{i j}\left(\delta_{j}^{k}-\nabla_{j} \mu^{k}\right)=-\epsilon^{i j}\left(\delta_{j}^{r}-\nabla_{j} \mu^{r}\right)\left(\psi^{-1}\right)_{r}^{k}, \\
& \sqrt{g^{(0)}} g_{(0)}^{i j}\left(\delta^{k}{ }_{j}-\nabla_{j} \mu_{d}^{k}\right)=-\epsilon^{i j}\left(\delta^{r}{ }_{j}-\nabla_{j} \mu^{r}\right)\left(\psi_{d}^{-1}\right)_{r}^{k},
\end{aligned}
$$

Multiplying equation (51) by $E_{k}$ and relation (52) by $B_{k}$, we arrive at the component of the gauge currents $J_{(F)}^{i}$, $J_{(B)}^{i}$, respectively. Having in mind that neglecting heat transport the gauge currents can be written as

$$
\begin{aligned}
& J_{(F)}^{i}=\sigma_{F F}^{i j} E_{j}+\sigma_{F B}^{i j} B_{j}, \\
& J_{(B)}^{i}=\sigma_{B F}^{i j} E_{j}+\sigma_{B B}^{i j} B_{j},
\end{aligned}
$$

we arrive at the conclusion that the DC conductivities constitute relations as follows:

$$
\begin{aligned}
\sigma_{F F}^{i j}=\epsilon^{i m} \psi_{m}^{j}, & \sigma_{F B}^{i j}=\frac{\alpha}{2} \epsilon^{i m} \psi_{m}^{j}, \\
\sigma_{B B}^{i j}=\epsilon^{i m}\left(\psi_{d}\right)^{j}{ }_{m}, & \sigma_{B F}^{i j}=\frac{\alpha}{2} \epsilon^{i m}\left(\psi_{d}\right)^{j}{ }_{m} .
\end{aligned}
$$

The inspection of the equations (45)-(46) and (51)-(52), we draw a conclusion that their consistency is ensured if $\psi_{a}{ }^{k} \psi_{k}{ }^{b}=-\delta_{a}{ }^{b}$ and similarly $\left(\psi_{d}\right)_{a}{ }^{k}\left(\psi_{d}\right)_{k}{ }^{b}=-\delta_{a}{ }^{b}$. On the other hand, the relations (55) and (566) enable us to 
write

$$
\begin{aligned}
& \operatorname{det} \sigma_{F F}=\frac{1}{2 !} \epsilon^{a b} \epsilon_{m k}\left(\sigma_{F F}\right)_{a}{ }^{m}\left(\sigma_{F F}\right)_{b}{ }^{k}=\frac{1}{2 !} \epsilon^{a b} \epsilon_{m k} \psi_{a}{ }^{m} \psi_{b}{ }^{k}, \\
& \operatorname{det} \sigma_{B B}=\frac{1}{2 !} \epsilon^{a b} \epsilon_{m k}\left(\sigma_{B B}\right)_{a}{ }^{m}\left(\sigma_{B B}\right)_{b}{ }^{k}=\frac{1}{2 !} \epsilon^{a b} \epsilon_{m k}\left(\psi_{d}\right)_{a}{ }^{m}\left(\psi_{d}\right)_{b}{ }^{k}, \\
& \operatorname{det} \sigma_{F B}=\frac{1}{2 !} \epsilon^{a b} \epsilon_{m k}\left(\sigma_{F F}\right)_{a}{ }^{m}\left(\sigma_{F F}\right)_{b}{ }^{k}=\frac{1}{2 !} \frac{\alpha^{2}}{4} \epsilon^{a b} \epsilon_{m k} \psi_{a}{ }^{m} \psi_{b}{ }^{k}, \\
& \operatorname{det} \sigma_{B F}=\frac{1}{2 !} \epsilon^{a b} \epsilon_{m k}\left(\sigma_{B B}\right)_{a}{ }^{m}\left(\sigma_{B B}\right)_{b}{ }^{k}=\frac{1}{2 !} \frac{\alpha^{2}}{4} \epsilon^{a b} \epsilon_{m k}\left(\psi_{d}\right)_{a}{ }^{m}\left(\psi_{d}\right)_{b}{ }^{k} .
\end{aligned}
$$

Having in mind the consistency condition, mentioned above, one arrives at the following:

$$
\operatorname{det} \sigma_{F F}=1, \quad \operatorname{det} \sigma_{B B}=1,
$$

which implies that

$$
\operatorname{det} \sigma_{F B}=\frac{\alpha^{2}}{4}, \quad \operatorname{det} \sigma_{B F}=\frac{\alpha^{2}}{4} .
$$

Consequently, for the determinant of the conductivity in the theory under consideration, we obtain

$$
\operatorname{det} \sigma=\left(\frac{1}{2 !}\right)^{2} \beta \epsilon_{b j_{1}} \epsilon_{m k} \epsilon^{d j_{2}} \epsilon_{s z} \psi_{b}^{m} \psi_{j_{1}}^{k}\left(\psi_{d}\right)^{s}{ }_{d}\left(\psi_{d}\right)_{j_{2}}^{z}=\beta \operatorname{det} \sigma_{F F} \operatorname{det} \sigma_{B B}=\beta
$$

where we set

$$
\beta=\tilde{\alpha}\left(1+\frac{\alpha^{2}}{4}\right)
$$

and $\tilde{\alpha}=1-\frac{\alpha^{2}}{4}$.

Let us assume that the conductivities $\sigma_{a b}, a, b=F, B$ do not depend on the spatial directions. Under these circumstances they can be considered as scalars. However, the full conductivity $\sigma$ of the system is the $2 \times 2$ matrix with entries $\left\{\sigma_{F F}, \sigma_{F B} ; \sigma_{B F}, \sigma_{B B}\right\}$ and thus on the basis of the equations (61) and (62) one has that det $\sigma=\sigma_{F F} \sigma_{B B}-$ $\sigma_{F B} \sigma_{B F}=1-(\alpha / 2)^{4}=\beta$. It can be seen that the considered bound is also valid for $\alpha=0$, when the matrix is diagonal $\sigma=\operatorname{diag}\left\{\sigma_{F F}, \sigma_{B B}\right\}$.

\section{CHARGED BLACK BRANE CASE}

In order to study the charged case, let us define for the adequate $U(1)$-gauge field, bulk dual tensors [44]

$$
\mathbb{F}^{r j}=-\frac{1}{2} \frac{\epsilon^{r j a b}}{\sqrt{-g}} F_{a b}, \quad \mathbb{B}^{r j}=-\frac{1}{2} \frac{\epsilon^{r j a b}}{\sqrt{-g}} B_{a b}
$$

with the property $\epsilon^{r t x y}=\frac{1}{\sqrt{-g}}$, as $r \rightarrow \infty$. It implies that one can find constant dual currents densities connected with Maxwell and auxiliary gauge fields, in the boundary theory, which imply

$$
I_{(F)}^{i}=\epsilon^{i j}\left(E_{j}+\frac{\alpha}{2} B_{j}\right), \quad I_{(B)}^{i}=\epsilon^{i j}\left(B_{j}+\frac{\alpha}{2} E_{j}\right) .
$$

The equations of motion $\partial_{i} J_{(F)}^{i}=0, \partial_{i} J_{(B)}^{i}=0$ yield that

$$
\begin{aligned}
& \partial_{r} \mathbb{E}\left[\mathbb{F}^{i r}+\frac{\alpha}{2} \mathbb{B}^{i r}\right]=0 \\
& \partial_{r} \mathbb{E}\left[\mathbb{B}^{i r}+\frac{\alpha}{2} \mathbb{F}^{i r}\right]=0
\end{aligned}
$$

where we have denoted the spatial average by $\mathbb{E}[M]=\frac{1}{L^{2}} \int d x^{2} M$, for the coordinates which satisfy the periodic boundary conditions $x_{i} \rightarrow x_{i}+L$. 
Just using the duals, at $r \rightarrow 0$, we get

$$
\begin{aligned}
& \mathbb{E}\left[\mathbb{F}^{i t}+\frac{\alpha}{2} \mathbb{B}^{i t}\right]=\epsilon^{i m} J_{(F) m}, \\
& \mathbb{E}\left[\mathbb{B}^{i t}+\frac{\alpha}{2} \mathbb{F}^{i t}\right]=\epsilon^{i m} J_{(B) m} .
\end{aligned}
$$

The spatial averaged dual electric currents connected with Maxwell and auxiliary fields, are independent on the radius of the bulk. It means that they can be defined on the black object event horizon

$$
\begin{aligned}
& \mathbb{E}\left[\mathbb{F}^{i t}+\frac{\alpha}{2} \mathbb{B}^{i t}\right]=\mathcal{E}_{j}+\frac{\alpha}{2} \mathcal{B}_{j}=\tau_{(F)}^{j k} I_{(F)_{k}}, \\
& \mathbb{E}\left[\mathbb{B}^{i t}+\frac{\alpha}{2} \mathbb{F}^{i t}\right]=\mathcal{B}_{j}+\frac{\alpha}{2} \mathcal{E}_{j}=\tau_{(B)}^{j k} I_{(B)_{k}},
\end{aligned}
$$

where $\tau_{(F)}^{j k}$ and $\tau_{(B)}^{j k}$ are the dual resistivity tensors bounded with Maxwell and hidden sector gauge fields. One can relate $\mathcal{E}_{i}, \mathcal{B}_{i}, I_{(F)}^{i}, I_{(B)}^{i}, J_{(F)}^{k}, J_{(B)}^{k}$ and obtain

$$
\begin{aligned}
& J_{(F) a}=\epsilon_{i a} \epsilon_{j k} \tau_{(F)}^{i j}\left(E^{k}+\frac{\alpha}{2} B^{k}\right), \\
& J_{(B) a}=\epsilon_{i a} \epsilon_{j k} \tau_{(B)}^{i j}\left(B^{k}+\frac{\alpha}{2} E^{k}\right) .
\end{aligned}
$$

It leads to the following relations:

$$
\begin{aligned}
& \sigma_{F F}^{i j}=\epsilon^{m i} \epsilon^{n j} \tau_{m n}^{(F)}, \\
& \sigma_{F B}^{i j}=\epsilon^{m i} \epsilon^{n j} \tau_{m n}^{(F)} \frac{\alpha}{2}, \\
& \sigma_{B B}^{i j}=\epsilon^{m i} \epsilon^{n j} \tau_{m n}^{(B)}, \\
& \sigma_{B F}^{i j}=\epsilon^{m i} \epsilon^{n j} \tau_{m n}^{(B)} \frac{\alpha}{2} .
\end{aligned}
$$

Consequently, it can be found that

$$
\begin{aligned}
\operatorname{det} \sigma_{F F} & =\frac{1}{2 !} \epsilon^{m i} \epsilon^{n j} \tau_{m n}^{(F)}, \\
\operatorname{det} \sigma_{F B} & =\frac{1}{2 !} \frac{\alpha^{2}}{4} \epsilon^{m i} \epsilon^{n j} \tau_{m n}^{(F)}, \\
\operatorname{det} \sigma_{B B} & =\frac{1}{2 !} \epsilon^{m i} \epsilon^{n j} \tau_{m n}^{(B)}, \\
\operatorname{det} \sigma_{B F} & =\frac{1}{2 !} \frac{\alpha^{2}}{4} \epsilon^{m i} \epsilon^{n j} \tau_{m n}^{(B)} .
\end{aligned}
$$

As in [8] we assume that the boundary theory constitutes the particle vortex dual, which leads to the conjecture that

$$
\operatorname{det} \sigma_{F F}=\frac{1}{\operatorname{det} \tau_{(F)}}, \quad \operatorname{det} \sigma_{B B}=\frac{1}{\operatorname{det} \tau_{(B)}},
$$

which in turn precedes to the conditions

$$
\left(\operatorname{det} \tau_{(F)}\right)^{2}=1, \quad\left(\operatorname{det} \tau_{(B)}\right)^{2}=1 .
$$

By virtue of the above relations, in the charge case the determinant of the conductivity is given by

$$
\operatorname{det} \sigma=\left(\frac{1}{2 !}\right)^{2} \beta_{1} \epsilon_{i_{1} k} \epsilon_{j_{1} l} \epsilon_{i_{2} a} \epsilon_{j_{2} b} \tau_{(F)}^{i_{1} j_{1}} \tau_{(F)}^{k l} \tau_{(B)}^{i_{2} j_{2}} \tau_{(B)}^{a b}=\beta_{1} \operatorname{det} \tau_{(F)} \operatorname{det} \tau_{(B)}=\beta_{1},
$$

where $\beta_{1}$ is given as follows:

$$
\beta_{1}=\tilde{\alpha}\left(1+\frac{\alpha^{2}}{4}\right) .
$$

The bound we have obtained in the charged case, is the same as in the uncharged model found earlier. The parameter $\beta_{1}$ is monotonously diminishing function of the coupling $\alpha$ from its canonical value 1 , when $\alpha \rightarrow 0$ and to the value 0 for $\alpha \rightarrow 2$. On physical grounds one expects $\alpha \leq 1$. The theory predicts the lowering of the bound from its the value 1 towards $\approx 0.94$ at $\alpha=1$. 


\section{VARIATIONAL ATTITUDE}

This section will be devoted to the variational techniques implemented in order to establish the lower bounds on DC-conductivities. The bounds will be achieved in an analogous way as the upper bounds of resistance of a disordered resistor network, based on the Thomson's principle [46]-47]. It states that if one runs a set of 'trial' currents through a resistor network, being subject to certain boundary conditions, the upper bound of the inverse conductivity can be computed by applying the variational principle to the power dissipated by the 'trial' currents in question. It happens that the power dissipated by 'trial' currents is minimal for the true distribution of the aforementioned currents.

To proceed further, let us recall that the Stokes equation on the black brane event horizon can be recast in the form as derived in Ref. [43]

$$
\begin{aligned}
\int \sqrt{g^{(0)}} d^{2} x\left[2 \nabla^{(i} v^{j)} \nabla_{(i} v_{j)}\right. & +\left(\nabla_{i} w+E_{i}\right)\left(\nabla^{i} w+E^{i}\right)+\left(\nabla_{i} w_{d}+B_{i}\right)\left(\nabla^{i} w_{d}+B^{i}\right) \\
\alpha\left(\nabla_{i} w\right. & \left.\left.+E_{i}\right)\left(\nabla^{i} w_{d}+B^{i}\right)+v^{m} \nabla_{m} \phi^{(0)} \nabla_{j} \phi^{(0)} v^{j}\right] \\
& =\int d^{2} x\left[Q^{i(0)} \xi_{i}+J_{(F)}^{i(0)} E_{i}+J_{(B)}^{i(0)} B_{i}\right],
\end{aligned}
$$

with the the adequate definitions of the currents, given by

$$
\begin{aligned}
J_{(F)}^{i(0)}=\left.J_{(F)}^{I}\right|_{\mathcal{H}} & =\sqrt{g^{(0)}} g_{(0)}^{i j}\left[\left(\nabla_{j}\left(\delta a_{t}^{(0)}\right)+E_{j}-a_{t}^{(0)} \delta g_{t j}^{(0)}\right)\right. \\
& \left.+\frac{\alpha}{2}\left(\nabla_{j}\left(\delta b_{t}^{(0)}\right)+B_{j}-b_{t}^{(0)} \delta g_{t j}^{(0)}\right)\right], \\
J_{(B)}^{i(0)}=\left.J_{(B)}^{I}\right|_{\mathcal{H}} & =\sqrt{g^{(0)}} g_{(0)}^{i j}\left[\left(\nabla_{j}\left(\delta b_{t}^{(0)}\right)+B_{j}-b_{t}^{(0)} \delta g_{t j}^{(0)}\right)\right. \\
& \left.+\frac{\alpha}{2}\left(\nabla_{j}\left(\delta a_{t}^{(0)}\right)+E_{j}-a_{t}^{(0)} \delta g_{t j}^{(0)}\right)\right], \\
Q^{i(0)}=\left.Q^{i}\right|_{\mathcal{H}} & =-4 \pi T \sqrt{g^{(0)}} g_{(0)}^{i j} \delta g_{t j}^{(0)} .
\end{aligned}
$$

Additionally one has that the following conservation relations are fulfilled:

$$
\nabla_{i} J_{(F)}^{i(0)}=0, \quad \nabla_{i} J_{(B)}^{i(0)}=0, \quad \nabla_{i} Q^{i(0)}=0 .
$$

Further, let us define

$$
J_{(F)}^{i(0)}=\sqrt{g^{(0)}} \mathcal{J}_{F}^{i}, \quad J_{(F)}^{i(0)}=\sqrt{g^{(0)}} \mathcal{J}_{F}^{i}, \quad Q^{i(0)}=4 \pi T \sqrt{g^{(0)}} v^{i} .
$$

Consequently the relation (87) may be rewritten in the form

$$
\begin{aligned}
\int d^{2} x & {\left[Q^{i(0)} \xi_{i}+J_{(F)}^{i(0)} E_{i}+J_{(B)}^{i(0)} B_{i}\right]=\int \sqrt{g^{(0)}} d^{2} x\left[2 \nabla^{(i} v^{j)} \nabla_{(i} v_{j)}+v^{m} \nabla_{m} \phi^{(0)} \nabla_{j} \phi^{(0)} v^{j}\right.} \\
+ & {\left[\frac{1}{\tilde{\alpha}}\left(\mathcal{J}_{F}^{i}-\frac{\alpha}{2} \mathcal{J}_{B}^{i}\right)-a_{t}^{(0)} v^{i}\right]\left[\frac{1}{\tilde{\alpha}}\left(\mathcal{J}_{i F}-\frac{\alpha}{2} \mathcal{J}_{i B}\right)-a_{t}^{(0)} v_{i}\right] } \\
+ & {\left[\frac{1}{\tilde{\alpha}}\left(\mathcal{J}_{B}^{i}-\frac{\alpha}{2} \mathcal{J}_{F}^{i}\right)-b_{t}^{(0)} v^{i}\right]\left[\frac{1}{\tilde{\alpha}}\left(\mathcal{J}_{i B}-\frac{\alpha}{2} \mathcal{J}_{i F}\right)-b_{t}^{(0)} v_{i}\right] } \\
+ & \left.\alpha\left[\frac{1}{\tilde{\alpha}}\left(\mathcal{J}_{F}^{i}-\frac{\alpha}{2} \mathcal{J}_{B}^{i}\right)-a_{t}^{(0)} v^{i}\right]\left[\frac{1}{\tilde{\alpha}}\left(\mathcal{J}_{i B}-\frac{\alpha}{2} \mathcal{J}_{i F}\right)-b_{t}^{(0)} v_{i}\right]\right],
\end{aligned}
$$

which is the subject of the following analysis.

\section{A. Bound on conductivities}

In order to establish the bounds on the conductivities in the holographic model of graphene, we shall analyze the left-hand side of the equation (93), which includes the definition of the dissipated power. The dissipated power will be provided by the following expression:

$$
P=J_{F}^{i} E_{i}+J_{B}^{i} B_{i}+Q^{i} \xi_{i}
$$


where the above quantities are normalized by averaging them spatially over the black brane event horizon, i.e.,

$$
J_{F}^{i}=\mathbb{E}\left[J_{(F)}^{i(0)}\right], \quad J_{B}^{i}=\mathbb{E}\left[J_{(B)}^{i(0)}\right], \quad Q^{i}=\mathbb{E}\left[Q^{i(0)}\right] .
$$

In what follows, we consider compact and flat spatial dimensions of the dual theory.

Using equation (93) the dissipative power (94) implies

$$
\begin{aligned}
P & =\mathbb{E}\left[2 \nabla^{(i} v^{j)} \nabla_{(i} v_{j}+v^{m} \nabla_{m} \phi^{(0)} \nabla_{j} \phi^{(0)} v^{j}\right. \\
& +\left[\frac{1}{\tilde{\alpha}}\left(\mathcal{J}_{F}^{i}-\frac{\alpha}{2} \mathcal{J}_{B}^{i}\right)-a_{t}^{(0)} v^{i}\right]\left[\frac{1}{\tilde{\alpha}}\left(\mathcal{J}_{i F}-\frac{\alpha}{2} \mathcal{J}_{i B}\right)-a_{t}^{(0)} v_{i}\right] \\
& +\left[\frac{1}{\tilde{\alpha}}\left(\mathcal{J}_{B}^{i}-\frac{\alpha}{2} \mathcal{J}_{F}^{i}\right)-b_{t}^{(0)} v^{i}\right]\left[\frac{1}{\tilde{\alpha}}\left(\mathcal{J}_{i B}-\frac{\alpha}{2} \mathcal{J}_{i F}\right)-b_{t}^{(0)} v_{i}\right] \\
& \left.+\alpha\left[\frac{1}{\tilde{\alpha}}\left(\mathcal{J}_{F}^{i}-\frac{\alpha}{2} \mathcal{J}_{B}^{i}\right)-a_{t}^{(0)} v^{i}\right]\left[\frac{1}{\tilde{\alpha}}\left(\mathcal{J}_{i B}-\frac{\alpha}{2} \mathcal{J}_{i F}\right)-b_{t}^{(0)} v_{i}\right]\right]
\end{aligned}
$$

One can consider $P$ as a functional of $v^{i}$ and the $U(1)$-gauge currents. It means that for arbitrary conserved periodic set of charge and heat currents directed along $v^{i}$ one has that

$$
\mathcal{J}_{F}^{i}=\tilde{\mathcal{J}}_{F}^{i}+\tilde{\tilde{\mathcal{J}}}_{F}^{i}, \quad \mathcal{J}_{B}^{i}=\tilde{\mathcal{J}}_{B}^{i}+\tilde{\tilde{J}}_{B}^{i}, \quad v^{i}=\tilde{v}^{i}+\tilde{\tilde{v}}^{i}
$$

where $\left(\tilde{v}^{i}, \tilde{\mathcal{J}}_{F}^{i}, \tilde{\mathcal{J}}_{B}^{i}\right)$ stands for the exact solution of the underlying system of hydrodynamical equations, being subject to the adequate boundary conditions. On the other hand, $\left(\tilde{\tilde{v}}^{i}, \tilde{\tilde{J}}_{F}^{i}, \tilde{\tilde{J}}_{B}^{i}\right)$ denote the deviations from the exact solution. Expansion of $P$ reveals that we get

$$
\begin{aligned}
P\left[v^{i}, \mathcal{J}_{F}^{i}, \mathcal{J}_{B}^{i}\right] & =P\left[\tilde{v}^{i}+\tilde{\tilde{v}}^{i}, \tilde{\mathcal{J}}_{F}^{i}+\tilde{\tilde{\mathcal{J}}}_{F}^{i}, \tilde{\mathcal{J}}_{B}^{i}+\tilde{\tilde{\mathcal{J}}}_{B}^{i}\right] \\
& =P\left[\tilde{v}^{i}, \tilde{\mathcal{J}}_{F}^{i}, \tilde{\mathcal{J}}_{B}^{i}\right]+P\left[\tilde{\tilde{v}}^{i}, \tilde{\tilde{J}}_{F}^{i}, \tilde{\tilde{J}}_{B}^{i}\right]+2 K,
\end{aligned}
$$

where the quantity $2 K$, implies

$$
\begin{aligned}
2 K & =2\left[\frac{1}{\tilde{\alpha}}\left(\tilde{\mathcal{J}}_{F}^{i}-\frac{\alpha}{2} \mathcal{J}_{B}^{i}\right)-a_{t}^{(0)} \tilde{v}^{i}\right]\left[\frac{1}{\tilde{\alpha}}\left(\tilde{\tilde{\mathcal{J}}}_{i F}-\frac{\alpha}{2} \tilde{\tilde{J}}_{i B}\right)-a_{t}^{(0)} \tilde{\tilde{v}}_{i}\right] \\
& +2\left[\frac{1}{\tilde{\alpha}}\left(\tilde{\mathcal{J}}_{B}^{i}-\frac{\alpha}{2} \mathcal{J}_{F}^{i}\right)-a_{t}^{(0)} \tilde{v}^{i}\right]\left[\frac{1}{\tilde{\alpha}}\left(\tilde{\tilde{J}}_{i B}-\frac{\alpha}{2} \tilde{\mathcal{J}}_{i F}\right)-a_{t}^{(0)} \tilde{\tilde{v}}_{i}\right] \\
& +\left[\frac{1}{\tilde{\alpha}}\left(\tilde{\mathcal{J}}_{F}^{i}-\frac{\alpha}{2} \mathcal{J}_{B}^{i}\right)-a_{t}^{(0)} \tilde{v}^{i}\right]\left[\frac{1}{\tilde{\alpha}}\left(\tilde{\tilde{\mathcal{J}}}_{i B}-\frac{\alpha}{2} \tilde{\tilde{J}}_{i F}\right)-a_{t}^{(0)} \tilde{\tilde{v}}_{i}\right] \\
& +\left[\frac{1}{\tilde{\alpha}}\left(\tilde{\mathcal{J}}_{B}^{i}-\frac{\alpha}{2} \mathcal{J}_{F}^{i}\right)-a_{t}^{(0)} \tilde{v}^{i}\right]\left[\frac{1}{\tilde{\alpha}}\left(\tilde{\tilde{\mathcal{J}}}_{i B}-\frac{\alpha}{2} \tilde{\mathcal{J}}_{i F}\right)-a_{t}^{(0)} \tilde{\tilde{v}}_{i}\right] \\
& +4 \nabla^{(i} \tilde{v}^{j)} \nabla_{(i} \tilde{\tilde{v}}_{j)}+2 \tilde{\tilde{v}}^{m} \nabla_{m} \phi^{(0)} \nabla_{i} \phi^{(0)} \tilde{v}^{i} .
\end{aligned}
$$

It can be shown using the current equations and integration by parts that $K=0$. Consequently, it reveals that

$$
P\left[v^{i}, \mathcal{J}_{F}^{i}, \mathcal{J}_{B}^{i}\right] \geq P\left[\tilde{v}^{i}, \tilde{\mathcal{J}}_{F}^{i}, \tilde{\mathcal{J}}_{B}^{i}\right]
$$

As was explained in [8], for the charged black brane one may set $v_{i}=0$, which trivially fulfils the constraints equations. Then we arrive at

$$
P\left[0, \mathcal{J}_{F}^{i}, \mathcal{J}_{B}^{i}\right]=\int \sqrt{g^{(0)}} d^{2} x \frac{\epsilon^{i_{1}}{ }_{a} \epsilon^{j_{1}} b}{\operatorname{det} \sigma}\left[\sigma_{i_{1} j_{1}}^{B B} \mathcal{J}_{F}^{a} \mathcal{J}_{F}^{b}+\sigma_{i_{1} j_{1}}^{F F} \mathcal{J}_{B}^{a} \mathcal{J}_{B}^{b}-\left(\sigma_{i_{1} j_{1}}^{F B}+\sigma_{i_{1} j_{1}}^{B F}\right) \mathcal{J}_{F}^{a} \mathcal{J}_{B}^{b}\right]
$$

On the other hand, using the equation (96), one arrives at the following:

$$
P\left[0, \mathcal{J}_{F}^{i}, \mathcal{J}_{B}^{i}\right]=\int \sqrt{g^{(0)}} d^{2} x\left[\frac{1}{\tilde{\alpha}}\left(\mathcal{J}_{F}^{i}\right)^{2}+\frac{1}{\tilde{\alpha}}\left(\mathcal{J}_{B}^{i}\right)^{2}-\frac{\alpha}{\tilde{\alpha}} \mathcal{J}_{F}^{i} \mathcal{J}_{i B}\right] .
$$

Comparison of the equations (101) and (102) give us the conditions imposed on the electrical conductivities, in general case. 
To commence with, let us analyze limits of the obtained relations. Firstly one supposes that in the absence of the heat current, we shall consider only the single current case. In this case $B_{i}=0, \alpha=0$ and the relations (53)-(54) reveal that

$$
\sigma_{B F}^{i j}=0, \quad \sigma_{F B}^{i j}=0, \quad \sigma_{B B}^{i j}=0
$$

and $E^{i}=\frac{\mathcal{J}_{F}^{i}}{\sigma_{F F}}$. Taking into account (153) and calculating the dissipative power we get

$$
P=\int \sqrt{g^{(0)}} d^{2} x \frac{\mathcal{J}_{F}^{i} \mathcal{J}_{i F}}{\sigma_{F F}}=\int \sqrt{g^{(0)}} d^{2} x \mathcal{J}_{F}^{i} \mathcal{J}_{i F}
$$

It implies that the following relation takes place:

$$
\sigma_{F F} \geq 1
$$

Consequently, for the model with only auxiliary $U(1)$-gauge field, one has that

$$
E_{i}=0, \quad \sigma_{F B}^{i j}=0,
$$

and $B_{i}=\frac{\mathcal{J}_{B}^{i}}{\sigma_{B B}}$. The same reasoning as above leads to the relation

$$
P=\int \sqrt{g^{(0)}} d^{2} x \frac{\mathcal{J}_{B}^{i} \mathcal{J}_{i B}}{\sigma_{B B}}=\frac{1}{\tilde{\alpha}} \int \sqrt{g^{(0)}} d^{2} x \mathcal{J}_{B}^{i} \mathcal{J}_{i B}
$$

and it yields that

$$
\sigma_{B B} \geq \tilde{\alpha}
$$

In the next step, because of the complexity of the exact relations, let us suppose that the existence only of $\mathcal{J}_{F}^{x}$ and $\mathcal{J}_{B}^{x}$ currents. By straightforward calculations it can be envisaged that $P\left[0, \mathcal{J}_{F}^{i}, \mathcal{J}_{B}^{i}\right]$ reduces to

$$
P\left[0, \mathcal{J}_{F}^{i}, \mathcal{J}_{B}^{i}\right]=\int \sqrt{g^{(0)}} d^{2} x \frac{1}{\operatorname{det} \sigma}\left[\sigma_{y y}^{B B}\left(\mathcal{J}_{F}^{x}\right)^{2}+\sigma_{y y}^{F F}\left(\mathcal{J}_{B}^{x}\right)^{2}-\left(\sigma_{y y}^{F B}+\sigma_{y y}^{B F}\right) \mathcal{J}_{F}^{x} \mathcal{J}_{B}^{x}\right] .
$$

Comparing the relations (109) and (102), the estimations for the adequate components of $\sigma_{\alpha \beta}^{i j}$ tensor can be achieved

$$
\frac{\sigma_{y y}^{B B}}{\operatorname{det} \sigma}=\frac{1}{\tilde{\alpha}}, \quad \frac{\sigma_{y y}^{F F}}{\operatorname{det} \sigma}=\frac{1}{\tilde{\alpha}}, \quad \frac{\sigma_{y y}^{F B}+\sigma_{y y}^{B F}}{\operatorname{det} \sigma}=\frac{\alpha}{\tilde{\alpha}} \text {. }
$$

\section{SUMMARY AND CONCLUSIONS}

In our paper we have studied the lower bounds of the electrical conductivities in the holographic model of the strongly interacting two-dimensional graphene sheet with disorder by means of the gauge-gravity duality. It happens that graphene close to the particle - hole symmetry point is a laboratory system fulfilling the strong coupling requirements. On the gravity side we elaborate the Einstein-Maxwell theory supplemented by the auxiliary $U(1)$-gauge field. The ordinary Maxwell and the auxiliary fields are coupled by the kinetic mixing term, with a coupling constant $\alpha$. In the studies we pay attention to the linear response of the black brane to the electric fields of the aforementioned gauge fields. On the field theory side, the situation coincides with the existence of two transport currents, which in graphene may correspond to electron and hole currents. The mixing parameter $\alpha$ may be responsible for the phase space constraints of scattering events in system with Dirac spectrum.

We have found the modifications of the bounds due to the coupling between the currents. For the physically expected values of $\alpha$-coupling constant which is smaller than 1 , the obtained bound $\beta$ for the conductivity tensor $\sigma$, $\operatorname{det} \sigma=\beta$ is only slightly below 1 .

It would be of interest to analyze the existence of the similar bound in Dirac or Weyl semimetals, being the three-dimensional analogues of graphene. 


\section{Acknowledgments}

KIW was partially supported by the grant DEC-2017/27/B/ST3/01911 of the National Science Center (Poland).

[1] P. W. Anderson, Absence of diffusion in certain random lattices, Phys. Rev. 109 (1958) 1492.

[2] N. F. Mott, Metal-Insulator Transition, Rev. Mod. Phys. 40, (1968) 677.

[3] D. Belitz and T. R. Kirkpatrick, The Anderson - Mott transition, Rev. Mod. Phys. 62, (1994) 261.

[4] S. V. Kravchenko, G. V. Kravchenko, J. E. Furneaux, V. M. Pudalov, and M. D'Iorio, Possible metal-insulator transition at $B=0$ in two dimensions, Phys. Rev. B 50 (1994) 8039.

[5] M. Yu. Melnikov, A. A. Shashkin, and V. T. Dolgopolov, Amy Y. X. Zhu and S. V. Kravchenko, S.-H. Huang and C. W. Liu, Quantum phase transition in ultrahigh mobility SiGe/Si/SiGe two-dimensional electron system, Phys. Rev. B 99 (2019) 081106.

[6] A. A. Shashkin and S. V. Kravchenko, Recent developments in the field of the metal-insulator transition in two dimensions, Appl. Sci. (Basel) 9, 1169 (2019).

[7] S. V. Kravchenko and M P Sarachik, Metal-insulator transition in two-dimensional electron systems, Rep. Prog. Phys. 67, 1 (2004).

[8] S. Grozdanov, A. Lucas, S. Sachdev, and K. Schalm, Absence of disorder-driven metal-insulator transitions in simple holographic models, Phys. Rev. Lett. 115 (2015) 221601.

[9] Andrew Lucas and Sean A. Hartnoll, Resistivity bound for hydrodynamic bad metals, Proc. Nat. Academy Sciences 114, (2017) 11344.

[10] R N Gurzhi, Hydrodynamic effects in solids at low temperature, Sov. Phys. Usp. 11 (1968) 255.

[11] L. W. Molenkamp and M. J. M. de Jong, Electron-electron-scattering-induced size effects in a two-dimensional wire, Phys. Rev. B 49 (1994) 5038.

[12] M. J. M. de Jong and L. W. Molenkamp, Hydrodynamic electron flow in high-mobility wires, Phys. Rev. B 51 (1995) 13389.

[13] C.Cao, E.Elliot, J.Joseph, H.Wu, J.Petricka, T.Schäfer, and J.E.Thomas, Universal quantum viscosity in a unitary Fermi gas, Science 331, (2011) 58.

[14] P. J. W. Moll, P. Kushwaha, N. Nandi, B. Schmidt, and A. P. Mackenzie, Evidence for hydrodynamic electron flow in PdCoO2, Science 351, (2016) 1061.

[15] D. A. Bandurin, I. Torre, R. Krishna Kumar, M. Ben Shalom, A. Tomadin, A. Principi, G. H. Auton, E. Khestanova, K. S. Novoselov, I. V. Grigorieva, L. A. Ponomarenko, A. K. Geim, and M. Polini, Negative local resistance caused by viscous electron backflow in graphene, Science 351, (2016) 1055.

[16] J. Crossno, J. K. Shi, K. Wang, X. Liu, A. Harzheim, A. Lucas, S. Sachdev, P. Kim, T. Taniguchi, K. Watanabe, T. A. Ohki, and K. C. Fong, Observation of the Dirac fluid and the breakdown of the Wiedemann-Franz law in graphene, Science 351, (2016) 1058.

[17] J. Zaanen, Electrons go with the flow in exotic material systems, Science 351, (2016) 1026.

[18] F. Ghahari, H-Y. Xie, T. Taniguchi, K. Watanabe, M.S. Foster, and P. Kim, Enhanced thermoelectric power in graphene: violation of the Mott relation by inelastic scattering, Phys. Rev. Lett. 116 (2016) 136802.

[19] R. Krishna Kumar, D. A. Bandurin, F. M. D. Pellegrino, Y. Cao, A. Principi, H. Guo, G. H. Auton, M. Ben Shalom, L. A. Ponomarenko, G. Falkovich, K. Watanabe, T. Taniguchi, I. V. Grigorieva, L. S. Levitov, M. Polini, and A. K. Geim, Superballistic flow of viscous electron fluid through graphene constrictions, Nature Physics 13, (2017) 1182.

[20] D. A. Bandurin, A.V. Shytov, L. S. Levitov, R. Krishna Kumar, A. I. Berdyugin, M. B. Shalom, I. V. Grigorieva, A. K. Geim, and G. Falkovich, Fluidity onset in graphene, Nature Commun. 9, (2018) 4533.

[21] B.N. Narozhny, I.V. Gornyi, A.D. Mirlin, and J. Schmalian, Hydrodynamic approach to electronic transport in graphene, Ann. Physik. (Leipzig) $\mathbf{5 2 9}$ (2017) 1700043.

[22] A. Lucas and K.C. Fong, Hydrodynamics of electrons in graphene, J. Phys.: Condens. Matter 30 (2018) 053001.

[23] M. Zarenia, A. Principi, and G. Vignale, Disorder-enabled hydrodynamics of charge and heat transport in monolayer graphene, 2D Mater. 6, 0350242019 (2019).

[24] The Reynolds number $R e$ is the ratio of the inertial to viscous forces and can be expressed as $R e=\frac{\rho u L}{\mu}$, where $\rho$ is fluid density, $u$ the velocity, $L$ the characteristic length affecting the flow and $\mu[\mathrm{Pa} \cdot \mathrm{s}]$ the dynamic viscosity.

[25] J. Mayzel, V. Steinberg, and A. Varshney, Stokes flow analogous to viscous electron current in graphene, Nature Commun. 10, (2019) 937.

[26] M. Müller, L. Fritz, and S. Sachdev, Quantum-critical relativistic magnetotransport in graphene, Phys. Rev. B 78 (2008) 115406.

[27] M. Müller, J. Schmalian, and L. Fritz, Graphene: a nearly perfect fluid, Phys. Rev. Lett. 103 (2009) 025301.

[28] A. Lucas, J. Crossno, K.C. Fong, P. Kim, and S. Sachdev, Transport in inhomogeneous quantum critical fluids and in the Dirac fluid in graphene, Phys. Rev. B 93 (2016) 075426.

[29] Y. Seo, G. Song, P. Kim, S. Sachdev, and S.-J. Sin, Holography of the Dirac fluid in graphene with two currents, Phys. Rev. Lett. 118 (2017) 036601.

[30] M. Rogatko and K.I. Wysokiński, Two interacting current model of holographic Dirac fluid in graphene, Phys. Rev. D $\mathbf{9 7}$ 
(2018) 024053.

[31] M. Rogatko and K.I. Wysokinski, Holographic calculation of the magneto-transport coefficients in Dirac semimetals, JHEP 01 (2018) 078.

[32] M.A.H. Vozmediano, M.I. Katsnelson, and F. Guine, Gauge fields in graphene, Phys. Reports 496 (2010) 109.

[33] G.E. Volovik and M.A. Zubkov, Emergent Horava gravity in graphene, Ann. Phys. 340 (2014) 352.

[34] A.Donos and J.P.Gauntlett, Navier-Stokes equations on black hole horizons and DC thermoelectric conductivity, Phys. Rev. D 92 (2015) 121901.

[35] E.Banks, A.Donos, and J.P.Gauntlett, Thermoelectric DC conductivities and Stokes flows on black hole horizons, JHEP $10(2015) 103$.

[36] S. Grozdanov, A. Lucas, and K. Schalm, Incoherent thermal transport from dirty black holes, Phys. Rev. D 93 (2016) 061901R.

[37] K.B. Fadafan, Conductivity bound from dirty black holes, Phys. Lett. B $\mathbf{7 6 2}$ (2016) 399.

[38] X.H. Ge, S. J. Sin, and S.F. Wu, Universality of DC electrical conductivity from holography, Phys. Lett. B 767 (2017) 63.

[39] T.N. Ikeda, A. Lucas, and Y. Nakai, Conductivity bounds in probe brane models, JHEP 04 (2016) 007.

[40] M. Baggioli and O. Pujalas, On holographic disorder-driven metal-insulator transitions, JHEP 01 (2017) 040.

[41] B. Gouteraux, E. Kiritsis, and W.J. Li, Effective holographic theories of momentum relaxation and violation of conductivity bound, JHEP 04 (2016) 122.

[42] B.S. Acharya, S.A.R. Ellis, G.L. Kane, B.D. Nelson, and M.J. Perry, Lightest visible-sector supersymmetric particle is likely unstable, Phys. Rev. Lett. 117 (2016) 181802.

[43] M. Rogatko, DC conductivities and Stokes flows in Dirac semimetals, hep-th 1910.04484 (2019).

[44] C.P. Herzog, P. Kovtun, S. Sachdev, and D.T. Son, Quantum critical transport, duality, and M-theory, Phys. Rev. D 75 (2007) 085020.

[45] A. Amoretti, A. Braggio, N. Maggiore, N. Magnoli, and D. Musso, Coexistence of two vector order parameters: a holographic model for ferromagnetic superconductivity, JHEP 01 (2014) 054.

[46] D.A. Levin, Y. Peres, and E.L. Wilmer, Markov chains and mixing times, Providence, RI: American Mathematical Society, 2009.

[47] A. Lucas, Hydrodynamic transport in strongly coupled disordered quantum field theories, New J. Phys. 17 (2015) 113007.

[48] A.V. Andreev, Steven A. Kivelson, and B. Spivak, Hydrodynamic Description of transport in strongly correlated electron systems, Phys. Rev. Lett. 106 (2011) 25804. 